\title{
Thermochemically-Informed Mass Transport Model for Zr in U-Zr Fuel
}

\section{Approved for public release. Distribution is unlimited.}

Max Poschmann Markus H.A. Piro Theodore M. Besmann Srdjan Simunovic

April 2020 


\section{DOCUMENT AVAILABILITY}

Reports produced after January 1, 1996, are generally available free via US Department of Energy (DOE) SciTech Connect.

Website: www.osti.gov/

Reports produced before January 1, 1996, may be purchased by members of the public from the following source:

National Technical Information Service

5285 Port Royal Road

Springfield, VA 22161

Telephone: 703-605-6000 (1-800-553-6847)

TDD: $703-487-4639$

Fax: 703-605-6900

E-mail: info@ntis.gov

Website: http://classic.ntis.gov/

Reports are available to DOE employees, DOE contractors, Energy Technology Data Exchange representatives, and International Nuclear Information System representatives from the following source:

Office of Scientific and Technical Information

PO Box 62

Oak Ridge, TN 37831

Telephone: 865-576-8401

Fax: 865-576-5728

E-mail: report@osti.gov

Website: http://www.osti.gov/contact.html

This report was prepared as an account of work sponsored by an agency of the United States Government. Neither the United States Government nor any agency thereof, nor any of their employees, makes any warranty, express or implied, or assumes any legal liability or responsibility for the accuracy, completeness, or usefulness of any information, apparatus, product, or process disclosed, or represents that its use would not infringe privately owned rights. Reference herein to any specific commercial product, process, or service by trade name, trademark, manufacturer, or otherwise, does not necessarily constitute or imply its endorsement, recommendation, or favoring by the United States Government or any agency thereof. The views and opinions of authors expressed herein do not necessarily state or reflect those of the United States Government or any agency thereof. 
Computational Sciences and Engineering Division

\title{
Thermochemically-Informed Mass Transport Model for Zr in U-Zr Fuel
}

\author{
Max Poschmann ${ }^{1}$, Markus H.A. Piro ${ }^{1}$, Theodore M. Besmann ${ }^{2}$, and Srdjan Simunovic ${ }^{3}$ \\ ${ }^{1}$ Ontario Tech University, Oshawa, ON, Canada \\ ${ }^{2}$ University of South Carolina, Columbia, SC \\ ${ }^{3}$ Oak Ridge National Laboratory, Oak Ridge, TN
}

Date Published: April 13, 2020

\author{
Prepared by \\ OAK RIDGE NATIONAL LABORATORY \\ Oak Ridge, TN 37831-6283 \\ managed by \\ UT-Battelle, LLC \\ for the \\ US DEPARTMENT OF ENERGY \\ under contract DE-AC05-00OR22725
}





\section{CONTENTS}

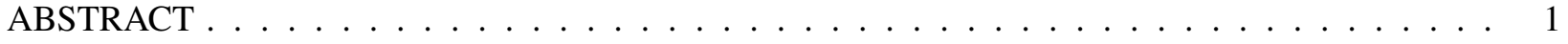

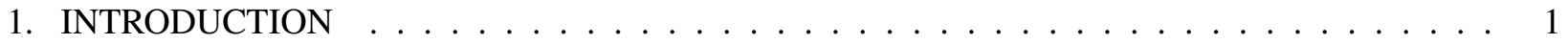

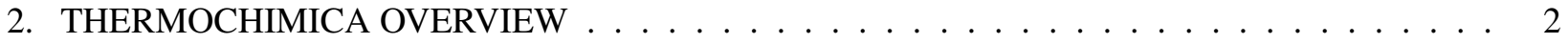

3. PREVIOUS SIMULATIONS OF ZIRCONIUM DIFFUSION IN METALLIC FUELS . . . . . . 3

4. BISON IMPLEMENTATION . . . . . . . . . . . . . . . . . . . . . . . . . . 4

4.1 ThermochimicaNodalUZr (MOOSE UserObject) . . . . . . . . . . . . . . . 4

4.2 UZrChemistryTransportSimplified (MOOSE AuxKernel) . . . . . . . . . . . . . 4

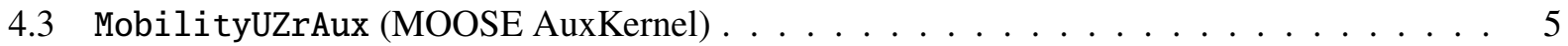

4.4 DiffusionGradChemPotentialMobilityAux (MOOSE Kernel) . . . . . . . . . . 5

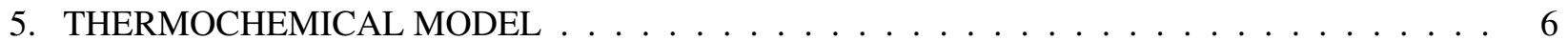

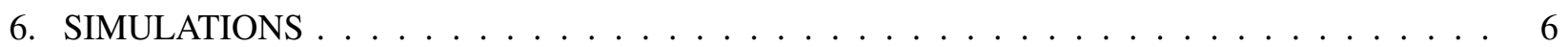

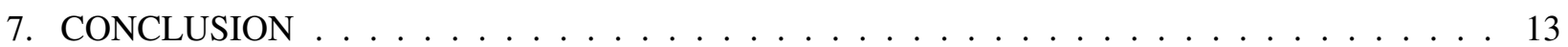

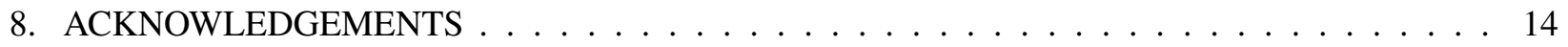

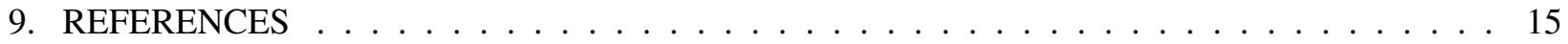




\begin{abstract}
Recent improvements to the coupled Thermochimica-MOOSE/BISON (Multi-physics Object-Oriented Simulation Environment) code system have enabled efficient calculations of species transport based on direct evaluation of composition and temperature dependent chemical potentials of the species. This presents an alternative to the traditional approach to species transport in nuclear fuels, which has been to employ a diffusion formulation that combines concentration-gradient driven Fickian diffusion with a Soret term based on a heat of transport fit to experimental data. Here we describe the application of the coupled code system to the diffusion of $\mathrm{Zr}$ in U-Zr metallic fuel. New classes implemented in BISON to solve this problem are documented. The $\mathrm{Zr}$ concentration profile after 50 years of diffusion is found to be strongly dependent on the assumptions made pertaining to how to mobility is calculated in multi-phase regions of the fuel element. Two assumptions are compared (simple averaging of mobilities and using the majority phase mobility), and good qualitative agreement with experimental measurements is obtained using the majority phase assumption.
\end{abstract}

\title{
1. INTRODUCTION
}

The open-source thermochemical library Thermochimica [29, 33] has previously been coupled with the Finite Element Method (FEM) Multi-physics Object-Oriented Simulation Environment (MOOSE) [10] fuel performance app BISON [13] for the purpose of providing thermodynamic information to simulations of nuclear fuels, such as phase fractions, chemical potentials, and concentrations of species, vacancies and interstitials.

Metallic fuels based on $\mathrm{U}-\mathrm{Zr}$ and $\mathrm{U}-\mathrm{Pu}-\mathrm{Zr}$ are receiving renewed interest as possible candidate fuels for generation IV reactor designs due to their high thermal conductivity, benign interactions with coolants such as sodium, good performance in accident scenarios, ability to make use of minor actinides recycled from other fuel, and the ability to reach relatively high burnup [4, 5]. However, constituent redistribution in these fuels under operation has continued to be an active and important area of research, as this can affect fuel performance and safety $[17,16,43,19,2,8,15,25]$.

A specific concern has been the redistribution of $\mathrm{Zr}$ in the fuel during operation, which can lead to Zr-depleted volumes in fuel elements with lower solidus temperatures, possibly causing local melting of the fuel $[24,4]$. In particular, experimental analysis has shown that $\mathrm{Zr}$ tends to be found in higher concentrations near the center, and to a lesser degree the periphery of the fuel [24, 16], with a Zr-depleted region in between where the solidus temperature is likely depressed. We have performed preliminary investigations into the diffusion of $\mathrm{Zr}$ in $\mathrm{U}-\mathrm{Zr}$ fuel with $10 \mathrm{wt} \%$ (22at\%) $\mathrm{Zr}$ using an updated Thermochimica-BISON implementation. These calculations have so far focused on $\mathrm{Zr}$ diffusion in a fixed temperature profile, and present a comparison between different assumptions about how mobility in two-phase regions should be treated. The generalized chemical potential gradient formulation of the driving force for diffusion employed here eliminates the need for a heat of transport term, reducing somewhat the number of parameters involved in the diffusion calculation.

In the following sections, we will provide a basic outline of Thermochimica operation and its incorporation into the MOOSE/BISON environment, describe the state of the literature on simulations of zirconium diffusion in metallic fuels, document the new BISON classes implemented in this work, and finally describe the simulations we have performed on this system. 


\section{THERMOCHIMICA OVERVIEW}

Thermochimica is a software library that determines the equilibrium combination of phases and compositions of those phases given input temperature, pressure, and concentrations of chemical elements. Thermochimica and the development of Thermochimica as well as its integration into the MOOSE/BISON framework have been well-documented in previous technical reports [29, 39, 38, 37, 40, 34], and journal articles [33, 30, 32, 31], as well as in the Thermochimica documentation (available on GitHub [26]).

From its genesis, Thermochimica has been designed to perform efficiently when coupled to multi-physics codes. This is reflected in the two-step algorithm design consisting of a leveling procedure followed by Gibbs Energy Minimization (GEM). The leveling solver, which is based on the algorithm of Eriksson and Thompson [7], efficiently provides an accurate initial estimate of the phase assemblage by ignoring the compositional dependence of the chemical potential of all solution species. All species and phases are therefore initially assumed to be stoichiometric phases. Quickly obtaining this estimate allows the GEM routine to begin much closer to the correct equilibrium phase assemblage and phase compositions than if it were initialized randomly; thus, a large number of unnecessary GEM iterations are reduced. With the implementation of a re-initialization algorithm documented in a previous report [34], this process is further accelerated by using the outcome of a previous calculation as the initial estimate for GEM optimization.

The approach of the GEM method is to optimize the system such that the residuals in mass balance equations and the minimization of total Gibbs energy are simultaneously minimized, subject to the constraints that the Gibbs Phase Rule is respected and conservation of mass [45, 33]. This leads to the Lagrangian function [42]:

$$
L=\frac{G}{R T}-\sum_{j=1}^{C} \tilde{\Gamma}_{j}\left(b_{j}-b_{j}^{m}\right)
$$

where $G$ is the total Gibbs energy, $R$ is the ideal gas constant, $T$ is the temperature, $C$ is the number of components* in the system, $\tilde{\Gamma}_{j}$ is the dimensionless chemical potential of component $j, b_{j}$ is the number of gram-atoms of component $j$, and $m$ is the GEM iteration index. Each GEM solver iteration requires the calculation of the Hessian (second partial derivative matrix) of $L$, which forms an $N \times N$ system where $N=C+\Phi$, where $\Phi$ is the total number of stable phases. This elucidates why the GEM solver is the most expensive component of Thermochimica as well as why calculation expense scales rapidly with the number of system components in the system.

As an equilibrium thermodynamics code, Thermochimica explicitly does not consider chemical kinetics, but rather assumes instantaneous local equilibrium. While this assumption is not strictly accurate, it is not a poor approximation for nuclear fuel applications because in these systems the temperature is typically high and chemical elements are well mixed due to the stochastic nature of nuclear fission. In multiphysics simulations involving Thermochimica and BISON, all kinetics are handled within BISON, and Thermochimica calculations consider only the instantaneous state of the system when invoked in a program. However, the kinetics are driven by variables provided by Thermochimica, such as the chemical potentials of diffusing species. This approach follows previous work in coupling Thermochimica with BISON for calculations of oxygen diffusion in nuclear fuels [39, 40, 34].

${ }^{*}$ The system components are typically taken as the chemical elements, but can be interpreted as integer combinations of the chemical elements. In the case of a system containing an ionic phase, the valence electron can be interpreted as a system component as well. 


\section{PREVIOUS SIMULATIONS OF ZIRCONIUM DIFFUSION IN METALLIC FUELS}

One of the central issues for $\mathrm{U}-\mathrm{Zr}$ metallic fuels is the tendency for redistribution of chemical elements. Fig. 1 illustrates such redistribution from a rare instance of post-irradiation analysis of the radial concentration gradients of uranium and zirconium for two Experimental Breeder Reactor-II (EBR-II) fuel pins experiencing 5at\% and 10at\% Fissions per Initial Metal Atom (FIMA) burnup [8, 15]. It is apparent that redistribution is restricted to approximately two-thirds of the radial distance from the centerline, with the likely explanation that the relatively cooler temperatures near the periphery of the fuel prevent sufficient migration energy for effective species transport. Such an assumption, however, is not obviously correct given the high thermal conductivity and small pin radius of the fuel, with estimated temperature gradients computed by Galloway et al. [9], for example, having a gradient at the higher range of $842-802^{\circ} \mathrm{C}$ and at the lower end of $777-747^{\circ} \mathrm{C}$. These $40^{\circ} \mathrm{C}$ and $30^{\circ} \mathrm{C} \Delta \mathrm{T}$ values, while certainly large enough to drive a composition gradient, would seem unlikely to include a very sharp decrease in mobility of the diffusive species as they are both small differences and span a uniform, single-phase region of the phase diagram (Fig. 2).
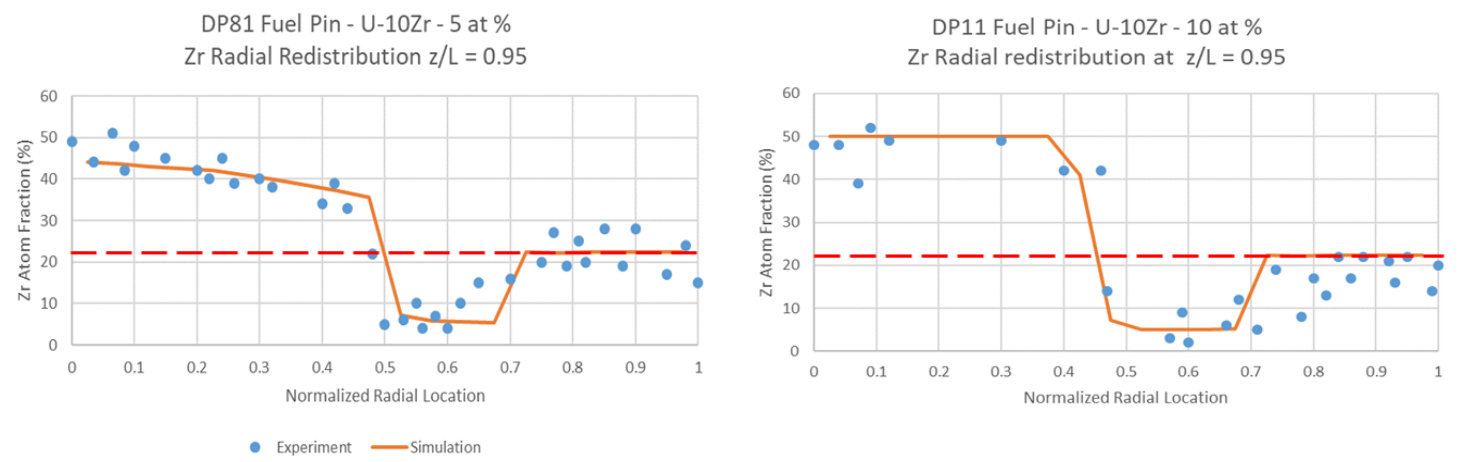

Figure 1. Measured $\mathrm{Zr}$ radial distribution in two irradiated EBR-II fuel pins experiencing 5 at $\%$ and 10 at $\%$ burnup. Also indicated are simulations of the elemental distribution based on empirical models, with the dashed red line indicating the original fuel's uniform $\mathrm{Zr}$ concentration. (courtesy A. Karahan, Argonne National Laboratory).

The computed temperatures of Galloway et al. [9] are in significant contrast to those assumed by Hirschhorn et al. [15], for which a radial gradient of $707-625^{\circ} \mathrm{C}$ is described. In this case, not only is the gradient twice as large, it also spans three different regions of the phase diagram (Fig. 2). An explanation tied to differing mobilities among the different phase structures can thus be argued to explain the unchanging fuel composition confined to the lower temperatures. Following the radial temperature profile of Hirschhorn et al. [15] it is apparent that redistribution between uranium and zirconium ceases below $660^{\circ} \mathrm{C}$, which corresponds to the phase boundary between the higher temperature two-phase region containing $\beta$-U $+\gamma-\mathrm{U}$ and the lower temperature $\alpha-\mathrm{U}+\gamma$-U region. If not solely related to temperature, the implication is that $\beta$-U may have a higher diffusivity for the metals than does $\alpha$-U. This is supported by experimental measurements of diffusivity in these phases $[36,1,18,16,28]$.

Hirschhorn et al. [15] have provided simulations using MARMOT, a mesoscale phase field code based on the MOOSE framework where the transport relations were optimized to the data recognizing the differing phase regions across the fuel pin radius. The result, with empirically fitted parameters, was relatively 
successful in reproducing the composition profiles.

These previous efforts to perform simulations of $\mathrm{Zr}$ redistribution in $\mathrm{U}-\mathrm{Zr}$ metallic fuels have employed a formulation of diffusion based on a term depending on the $\mathrm{Zr}$ concentration gradient, plus a Soret term corresponding to the temperature gradient $[16,8,15]$. In previous work on oxide fuels, we have developed tools that combine these terms into a generalized chemical potential formulation, in which the gradient of the chemical potential (depending on both concentration and temperature, and calculated by Thermochimica) is taken as the driving force for diffusion [41]. In this work, we extend these methods to metallic fuel systems, employing detailed thermochemical calculations to determine key parameters on the fly for our finite element diffusion calculation.

\section{BISON IMPLEMENTATION}

The coupling of Thermochimica with BISON for $\mathrm{Zr}$ transport in metallic fuel builds upon previous efforts to couple Thermochimica calculations into the MOOSE framework [39, 38, 37, 40, 34], including the use of the re-initialization algorithm [34]. The classes implemented to complete this work also build on those implemented in those previous efforts. In particular, ThermochimicaNodalUZr is a child class of ThermochimicaNodalData that parallels the existing ThermochimicaNodalU02X, and UZrChemistryTransportSimplified is a child class of ThermochimicaAux that parallels U02XChemistryTransportSimplified. New classes MobilityUZrAux and DiffusionGradChemPotentialMobilityAux were also implemented to handle mobility calculations at nodes through the use of an AuxKernel, replacing the previous implementation of mobility as a Material found in MobilityU02X.

\subsection{ThermochimicaNodalUZr (MOOSE UserObject)}

The ThermochimicaNodalUZr UserObject is a specialization of the ThermochimicaNodalData UserObject implemented previously, designed to perform calculations for the U-Zr system. This UserObject makes use of the PresetElementMass function in the Thermochimica interface to overwrite the mass of zirconium from the elements AuxVariable used in the base ThermochimicaNodalData with the value in the zirconium variable. The Thermochimica calculation is performed by the base class, and then Thermochimica outputs of interest are extracted via various accessor functions provided by the Thermochimica interface. These are then saved in member variables and made available to other objects via get- accessor functions.

\subsection{UZrChemistryTransportSimplified (MOOSE AuxKernel)}

UZrChemistryTransportSimplified uses results from Thermochimica calculations to calculate values of AuxVariables. In this particular case, ThermochimicaNodalUZr is a UserObject that extracts data such as $\alpha, \beta$, and $\gamma$ phase fractions (and the concentration of $\mathrm{Zr}$ in these phases), and zirconium chemical potential from a Thermochimica calculation. This AuxKernel copies that data to the relevant AuxVariables, which are later used to calculate the driving force and mobility for $\mathrm{Zr}$ diffusion within $\mathrm{U}-\mathrm{Zr}$ fuel. 


\subsection{MobilityUZrAux (MOOSE AuxKernel)}

This AuxKernel is an implementation of a mobility calculation as an AuxKernel rather than as a Material as in MobilityU02X. The purpose is to provide mobilities directly at the nodes such that they can be interpolated into the element, rather than calculate the mobility based on interpolated compositions. This makes the use of properties derived from thermochemical calculations more uniform, and allows for control over evaluation of the mobilities. This AuxKernel is for use in conjunction with the DiffusionGradChemPotentialMobilityAux Kernel described in Section 4.4.

This function calculates the mobility parameter for zirconium in metallic fuel based on the work of [8] by default, but other parameters may be supplied as arguments. It uses zirconium fractions of alpha, beta, and gamma phases as calculated in UZrChemistryTransportSimplified. It also couples the temperature field from the heat diffusion calculation, if performed.

Currently, the majority phase is taken to dominate the mobility at each evaluation. This was modified to allow for other assumptions to be tested in the work presented here.

Note that this AuxKernel requires inputs of both phases and species of interest to output concentrations. In particular, the phases ORTHORHOMBIC_A20, TETRAGONAL_U, BCC_A2_1, and BCC_A2_2 and the ZR species in each must be included in output_phases and output_species, respectively.

\subsection{DiffusionGradChemPotentialMobilityAux (MOOSE Kernel)}

This Kernel is based on, and very similar to, the Kernel DiffusionGradChemPotential described in detail in Ref. [40]. In this case the species mobility is provided by an AuxKernel such as MobilityUZrAux that is evaluated at the nodes, rather than from a Material. Some details will be provided here so that this modification can be explained, but a complete description of the Kernel implementation will not be recapitulated.

As in DiffusionGradChemPotential, this Kernel drives mass diffusion based on a mechanistic description of the diffusion process $[20,6]$. Using the volumetric density of a component, $n_{k}$, as the primary variable with units of $\left[\mathrm{mol} / \mathrm{m}^{3}\right]$, the flux is defined as the amount of component transported across a unit area normal to the flux direction, over a unit of time. Assuming that the diffusing component can be assigned an average constant drift velocity, $v_{k}$, its molar flux, $J_{k}$, is:

$$
J_{k}=v_{k} n_{k}
$$

where $J_{k}$ has units of $\mathrm{mol} / \mathrm{m}^{2} \mathrm{~s}$, and $v_{k}$ has units of $\mathrm{m} / \mathrm{s}$. Using a linear approximation for uncorrelated movements of transporting particles, the drift velocity is a result of the product of the driving force, $F_{k}$, imparted to a particle, and the proportionality factor, $M_{k}$ :

$$
v_{k}=M_{k} F_{k}
$$

The proportionality factor $M_{k}$, termed mechanical mobility, is an inverse of the resistance experienced by a particle as it interacts with its environment. This is related to the diffusivity $D$ by the Einstein relation:

$$
M=\frac{D}{R T}
$$


and $D$ in turn is modeled by the exponential expression:

$$
D=D_{0} e^{-\frac{Q}{R T}}
$$

with $D_{0}$ and $Q$ model parameters.

The driving force is taken to be the gradient of the chemical potential:

$$
F_{k}=-\nabla \mu_{k}
$$

which results in the mass flux equation:

$$
J_{k}=M_{k} n_{k} F_{k}=-M_{k} n_{k} \nabla \mu_{k}
$$

The conservation equation for implementation in a FEM solver can be written as:

$$
\frac{\partial n_{k}}{\partial t}+\nabla \cdot\left(-M_{k} n_{k} \nabla \mu_{g k}\right)=0
$$

In this implementation of the diffusion Kernel, the mobility is supplied by an AuxVariable that has been calculated at the nodes by an AuxKernel (MobilityUZrAux), and is interpolated to the element interior within the Kernel. An AuxKernel (UZrChemistryTransportSimplified) also provides the chemical potential of $\mathrm{Zr}$ at every node, which allows for the evaluation of the gradient by this Kernel. Both of these AuxKernels make use of data stored in the UserObject ThermochimicaNodalUZr, which is the class used to setup and make calls to Thermochimica. The remainder of the implementation is identical to that of DiffusionGradChemPotential, and details may be found in Ref. [40].

\section{THERMOCHEMICAL MODEL}

The U-Zr fuel is the base binary system, with current thermodynamic models and values derived from the work of Quaini et al. [35] who reassessed existing results to ameliorate issues in the extrapolation to the $\mathrm{U}-\mathrm{Zr}-\mathrm{O}$ system. As noted in our previous efforts and repeated here, the descriptions of the body-centered cubic (BCC) solid solution $\gamma-\mathrm{U} / \beta-\mathrm{Zr}$ as well as the $\delta$-(U, Zr) were reassessed by Quaini et al. [35] and used in the current Thermodynamics for Advanced Fuels-International Database (TAF-ID) [44]. Both the BCC $\gamma$-U/ $\beta$-Zr and the $\delta$-(U, Zr) phases play an important role in $\mathrm{U}-\mathrm{Zr}$ fuel. The BCC solid solution also extends to U-Pu-Zr systems with $\mathrm{Cr}$ [3], Mo [27], $\mathrm{Nb}[12,23], \mathrm{Np}$ [22], Ta, Ti [21], and W, as FPs with varying solubility.

The U-Zr nuclear fuel system has been assessed, with the phase equilibria accurately reproduced from thermochemical models and values derived from TAF-ID [44, 11, 35]. Fig. 2 is a resulting computed phase diagram. Also indicated on the diagram is the typical fuel alloy composition, U-10wt\% Zr, or U-22at\% Zr, that was utilized in our simulations as well as in reported experimental irradiations in both EBR-II [16], and most recently included in rodlets irradiated in the Advanced Test Reactor (ATR) [14].

\section{SIMULATIONS}




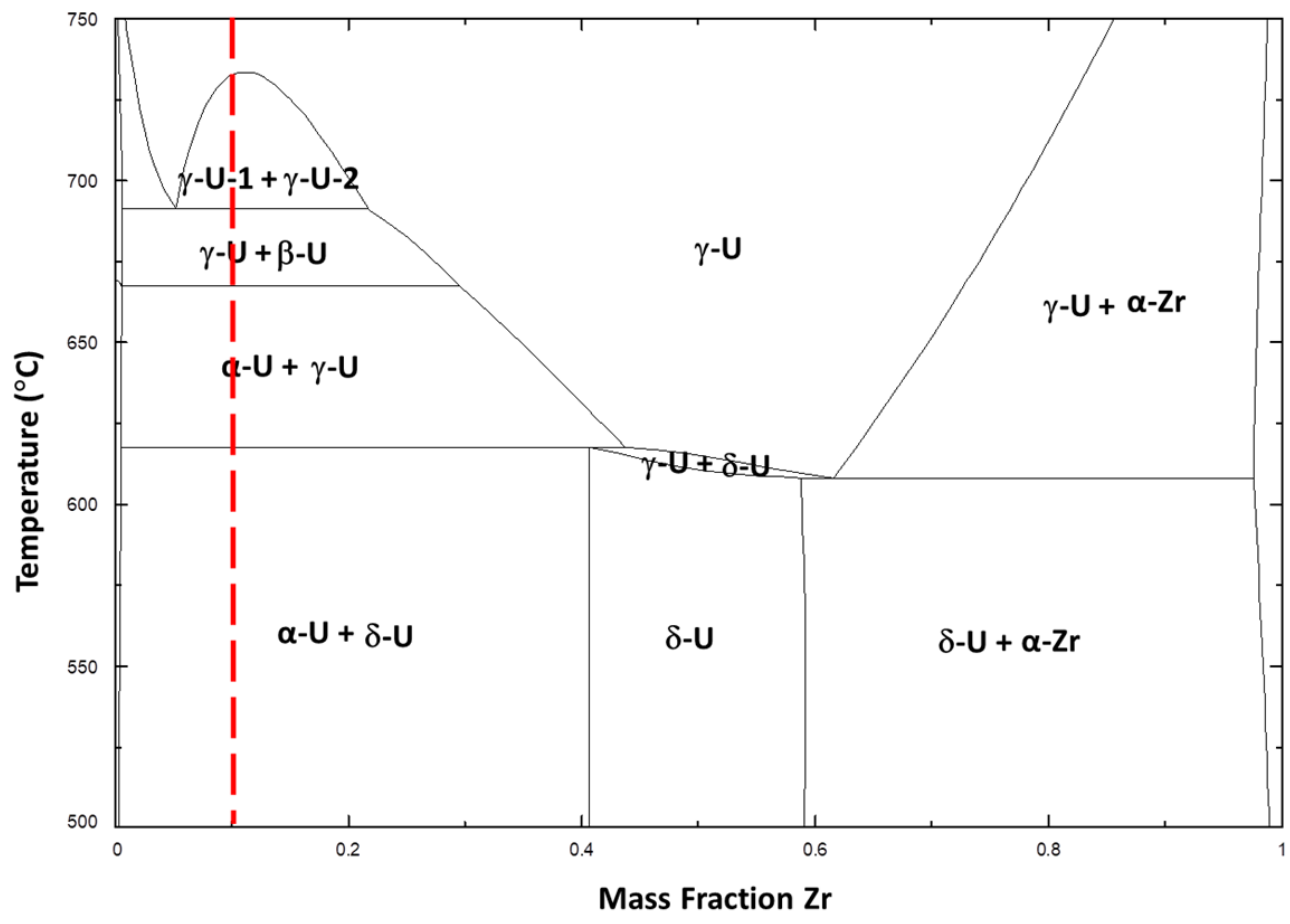

Figure 2. U-Zr phase diagram based on TAF-ID models and values, derived from Quaini et al. [35]. Vertical, red dashed line indicates fuel composition U-22at\%Zr (U-10wt\%Zr).

While in our previous work involving oxide fuels, the $\mathrm{UO}_{2 \pm x}$ fluorite phase is the dominant phase in all regions of the fuel element throughout the simulation, the case for metallic fuel is not as simple. Over the temperature range of interest (approximately $895 \mathrm{~K}$ to $980 \mathrm{~K}$ [15]) the initial fuel composition falls in three different phase stability regions: in the zone nearest the core - where temperature is highest - the fuel is in a miscibility gap in the $\gamma$ phase; in the intermediate zone, the fuel is in a 2-phase $\gamma+\beta$ region; and near the periphery the fuel is in a 2-phase $\gamma+\alpha$ region [16]. The phase fractions computed by Thermochimica for the initial fuel composition are show in Fig. 3, and the $\mathrm{Zr}$ concentration in each phase is shown in Fig. 4. While different mobility rates for $\mathrm{Zr}$ in these three phases $(\alpha, \beta$, and $\gamma)$ have been proposed $[16,19,8]$, in general it is observed that the mobility of $\mathrm{Zr}$ in the $\gamma$ phase is much faster than in either the $\alpha$ or $\beta$ phases. This makes the question of how to treat diffusion in 2-phase regions crucial to the overall prediction of $\mathrm{Zr}$ diffusion. While the most accurate treatment of this problem would incorporate explicitly the microstructure of the fuel, this would be extremely computationally demanding.

Instead we have implemented two different models for how mobility should be calculated in 2-phase regions. We demonstrate that the results derived from these two treatments are significantly different, and that one of the choices qualitatively reproduces experimental observations with respect to the three $\mathrm{Zr}$ concentration zones. The two mobility models implemented are a simple mixing model in which the total mobility is the average of the mobilities of present phases weighted by their phase fractions, and a model in which the majority phase is taken to dominate the mobility (similar to the discontinuation of $\mathrm{Zr}$ flux through minority phases used by Hofman et al. [16]). The second model is based on the assumption that a phase with greater than $50 \%$ volume fraction will be continuous across the domain. The resulting $\mathrm{Zr}$ concentration profiles after 50 years of simulation for both models are shown in Fig. 5. This extremely 


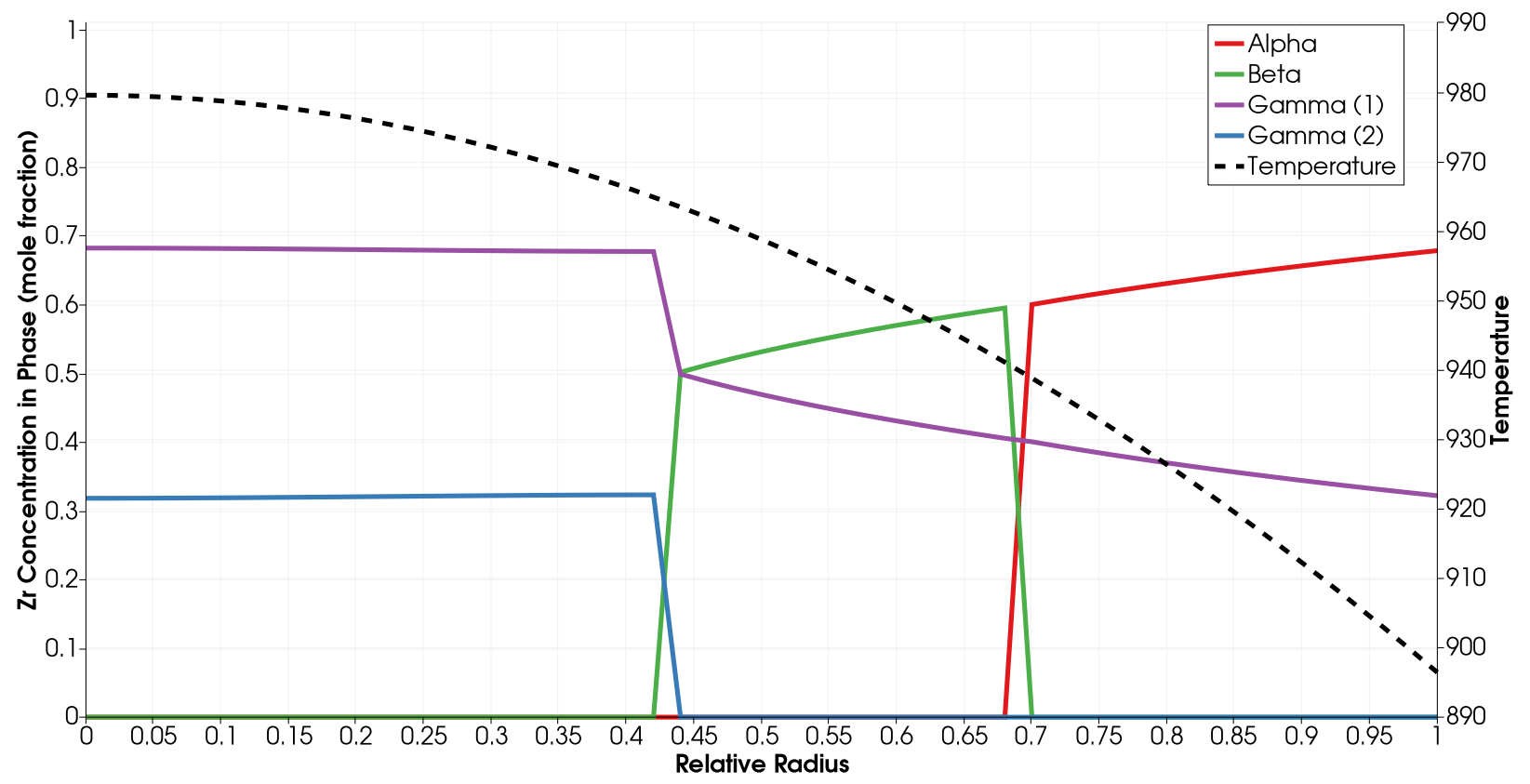

Figure 3. Phase fractions for the initial $\mathrm{U}-\mathrm{Zr}$ fuel composition of uniform $22 \mathrm{at} \% \mathrm{Zr}$ under the imposed temperature profile. In agreement with the phase diagram shown in Fig. 2, the fuel element displays three different phase stability regions. From the interior (highest temperature) to the exterior (lowest temperature) of the element, these are a $\gamma$-phase miscibility gap, a $\gamma+\beta$ mixture, and a $\gamma+\alpha$ mixture.

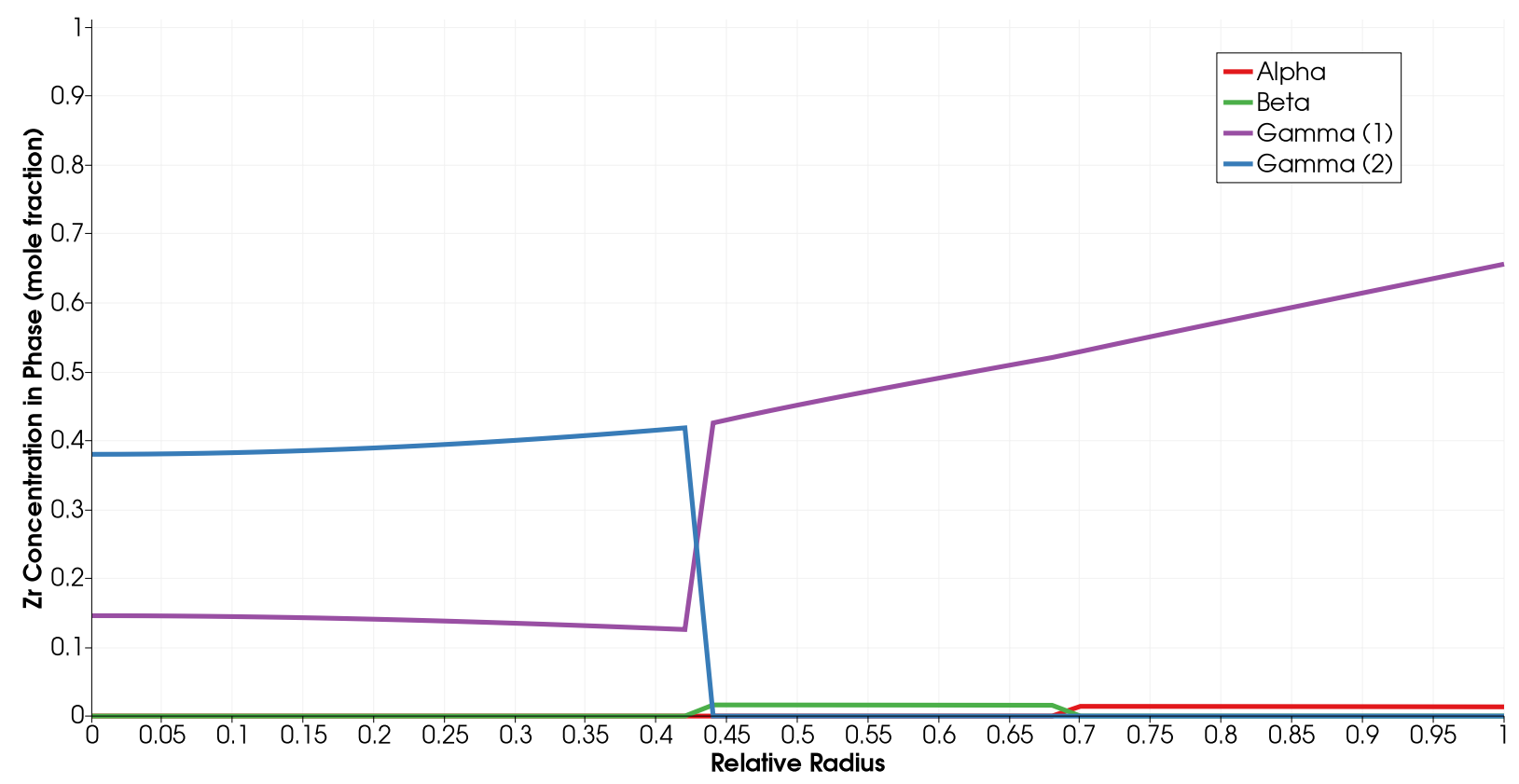

Figure 4. $\mathrm{Zr}$ concentration in each phase for the initial $\mathrm{U}-\mathrm{Zr}$ fuel composition of uniform $22 \mathrm{at} \% \mathrm{Zr}$ under the imposed temperature profile. 


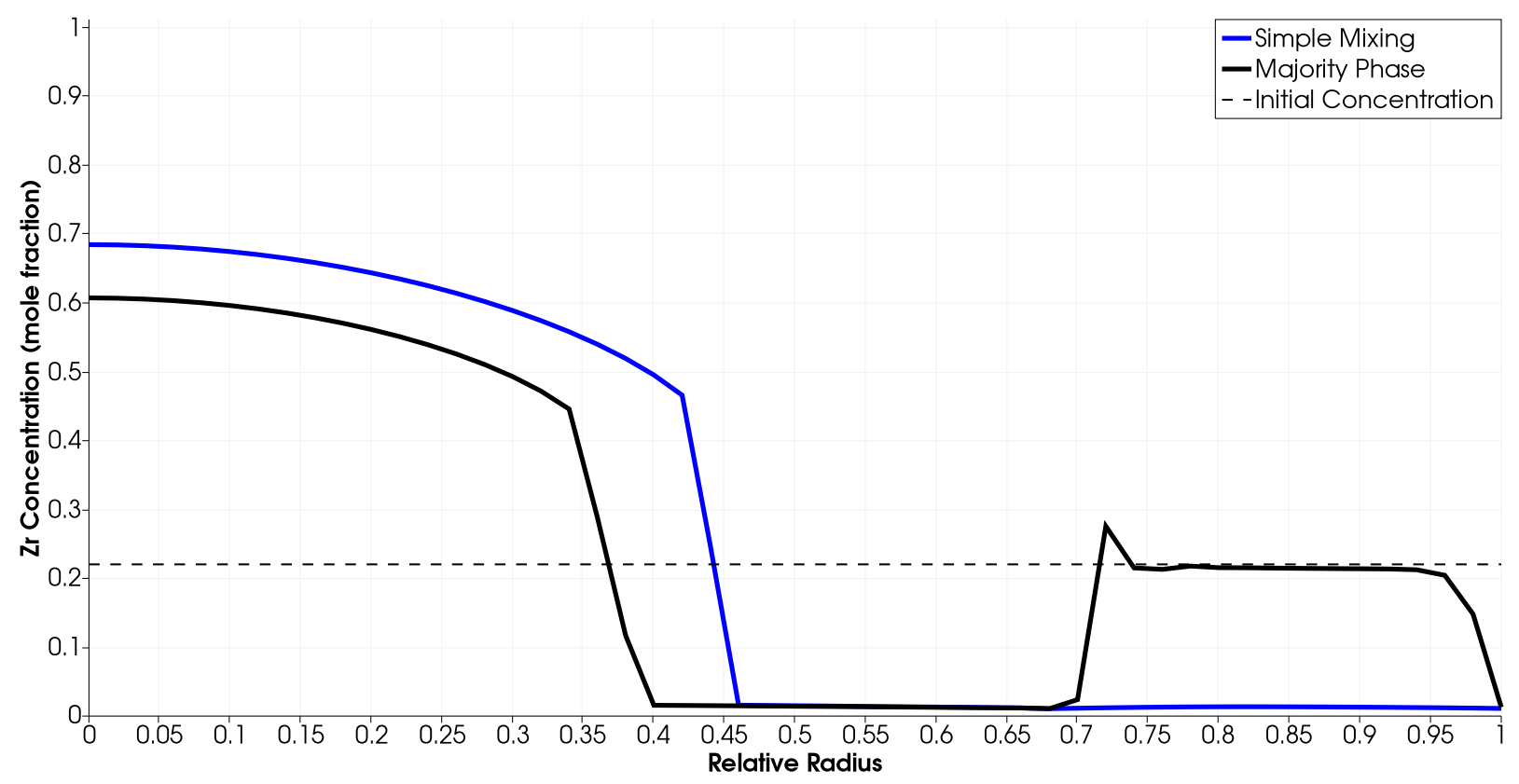

Figure 5 . $\mathrm{Zr}$ concentration profiles after 50 years of diffusion using the simple mixing mobility model and the majority phase mobility model.

long time domain was employed so that the steady-state-like tendencies of the mixing models could be demonstrated. In both cases, the finite element model of the fuel pellet was represented by a 1D radial mesh with 50 elements of equal size. The use of a mass-lumped time derivative was found to significantly improve the numerical stability of these calculations. The temperature profile of Hirschhorn et al. [15] was assumed, along with the diffusion coefficients listed in Table 1. Note that in this work we are not attempting to optimize these coefficients, but rather to demonstrate our method using the generalized chemical potential and the effects of assumptions about mobility in two-phase regions. As the generalized chemical potential method supersedes the need for Soret terms, the Soret coefficients (heats of transport) from Ref. [8] are neglected here.

Table 1. Diffusivity parameters employed in this study.

\begin{tabular}{cccc}
\hline Phase & $D_{0}\left[\mathrm{~m}^{2} / \mathrm{s}\right]$ & $Q[\mathrm{~kJ} / \mathrm{mol}]$ & Ref. \\
\hline$\alpha$ & $2.0 \times 10^{-7}$ & 170 & {$[16,15]$} \\
$\beta$ & $5.7 \times 10^{-5}$ & 180 & {$[16,15]$} \\
$\gamma$ & $10^{-5.1-8.05 x_{Z r}+9.13 x_{Z r}^{2}}$ & $128-107 x_{Z r}+174 x_{Z r}^{2}$ & {$[16,8]$} \\
\hline
\end{tabular}

The mobility of zirconium at the beginning of both simulations is shown in Fig. 6 . In the simple mixing model for mobility, the much-higher $\gamma$ phase mobility dominates in all regions of the fuel element, and thus the $\mathrm{Zr}$ mobility is relatively high across the entire element. This allows all of the $\mathrm{Zr}$ to diffuse down the chemical potential gradient and into the warmer core region. However, in the simulation using the majority phase mobility approximation, the mobility of $\mathrm{Zr}$ in the region near the periphery is significantly reduced, and it is also reduced to a lesser degree in the intermediate region. This results in a $\mathrm{Zr}$ concentration profile 


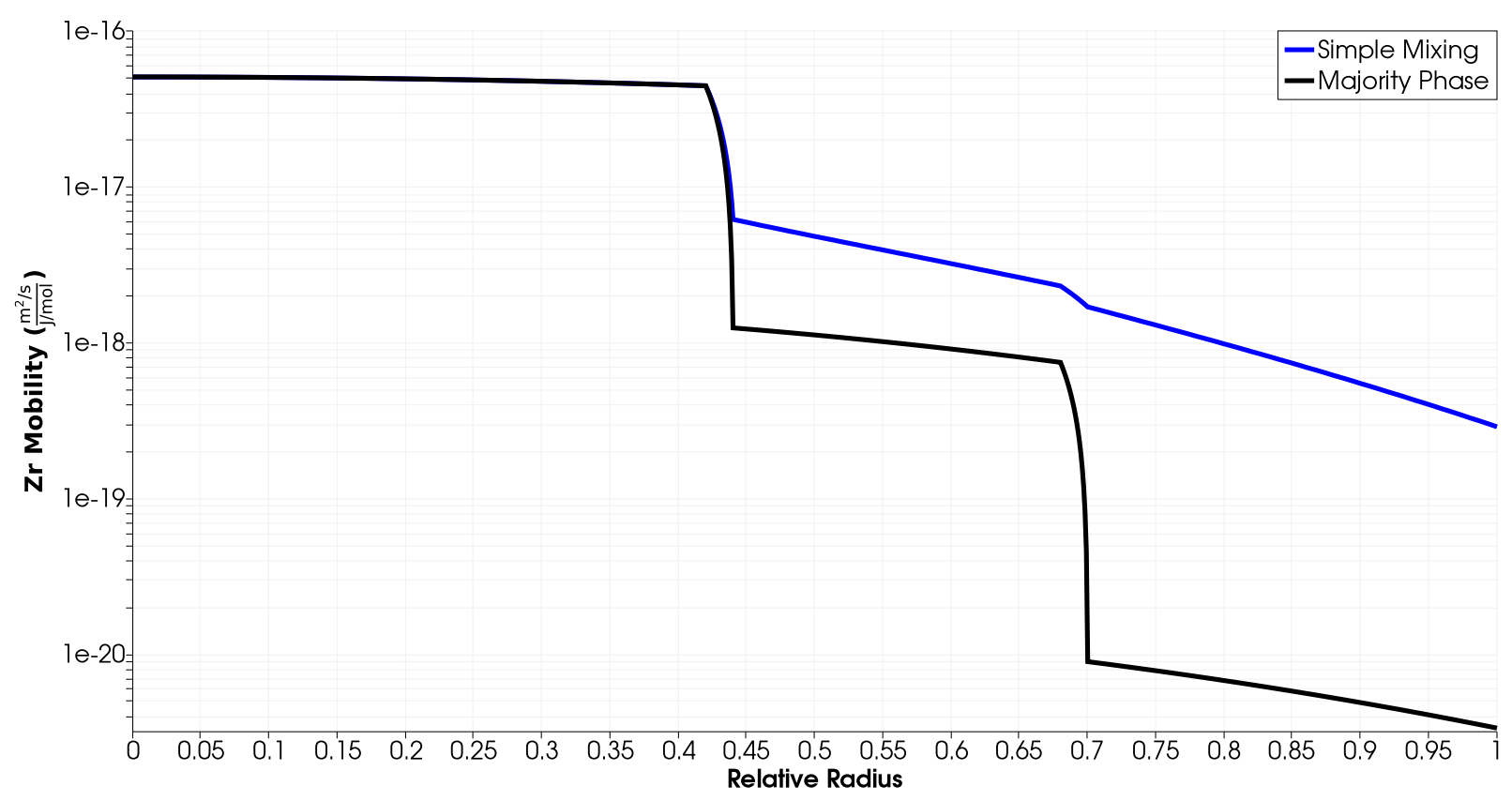

Figure 6. $\mathrm{Zr}$ mobility for the initial $22 \mathrm{at} \% \mathrm{Zr}$ fuel element, as calculated by the simple mixing mobility model and the majority phase mobility model. Note that because the mobility of $\mathrm{Zr}$ in the $\gamma$ phase is strongly dependent on the $\mathrm{Zr}$ concentration in that phase, there are discontinuities in mobility even when the simple mixing model is employed.

that looks much more similar to previous experimental results. In this simulation it is the interplay between chemical potential gradient (moving $\mathrm{Zr}$ towards the core) and the varying mobility in the different phase stability regions of the fuel element that lead to the $\mathrm{Zr}$-depleted intermediate region. However, we find no melting in this region for the applied temperature profile. If the $\mathrm{Zr}$ depletion results in significant change to the thermal conductivity this temperature profile may change during operation and affect this result.

The phase fractions resulting from each mixing model are shown in Figs. 7-8, and the corresponding $\mathrm{Zr}$ concentrations in each phase after 50 years of diffusion are shown in Figs. 9-10.

Fig. 7 shows that for the simple mixing case, after 50 years diffusion has created three single-phase regions where there were originally three two-phase regions. These are now $\gamma, \beta$, and $\alpha$ from the element interior to exterior, and the phase boundaries are in approximately the same locations as the original boundaries between two-phase regions. For the majority phase rule calculation, however, significant two-phase regions still exist after 50 years, in particular the $\gamma-\alpha$ region near the element surface (Fig. 8).

The $\mathrm{Zr}$ mobilities resulting from each model after 50 years of diffusion are shown in Fig. 11. The final values are similar, as there are no longer any two-phase regions remaining in the simple mixing case, and thus the calculated mobility tends to agree with that from the majority phase case. 


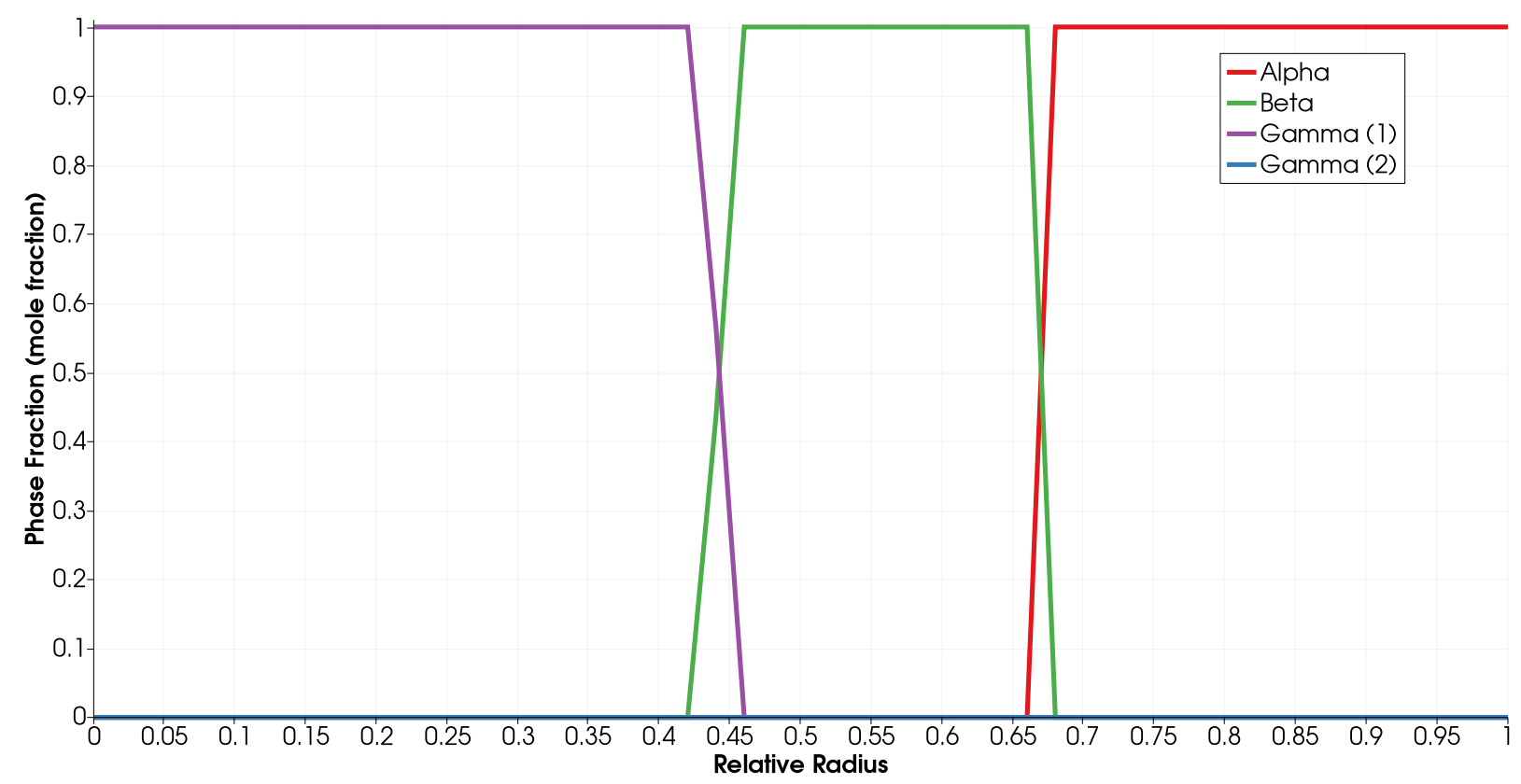

Figure 7. Phase fractions for the U-Zr fuel after 50 years of diffusion under the imposed temperature profile with the simple mixing rule applied to two-phase regions.

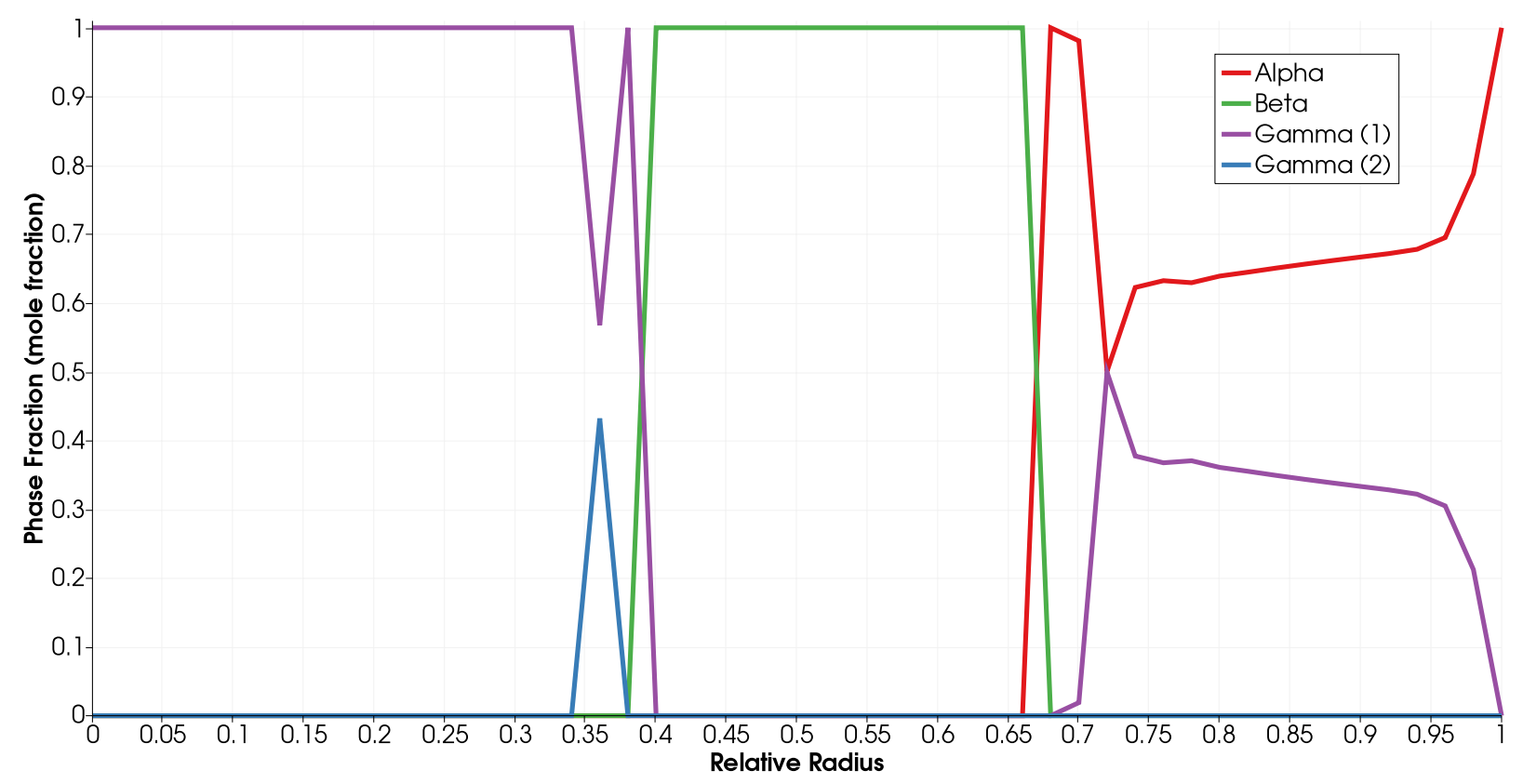

Figure 8. Phase fractions for the U-Zr fuel after 50 years of diffusion under the imposed temperature profile with the majority phase rule applied to two-phase regions. 


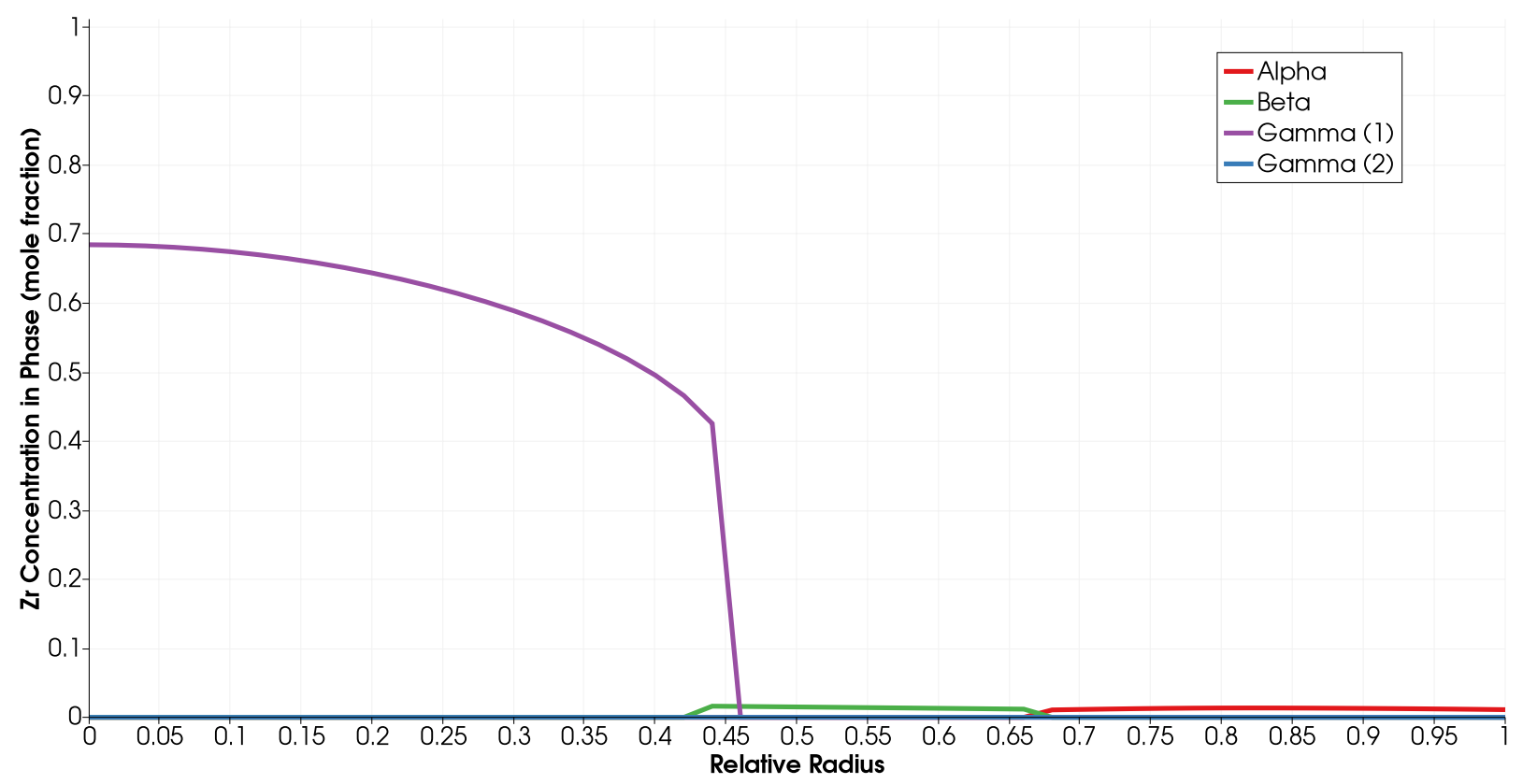

Figure 9. $\mathrm{Zr}$ concentration in each phase for the $\mathrm{U}-\mathrm{Zr}$ fuel after 50 years of diffusion under the imposed temperature profile with the simple mixing rule applied to two-phase regions.

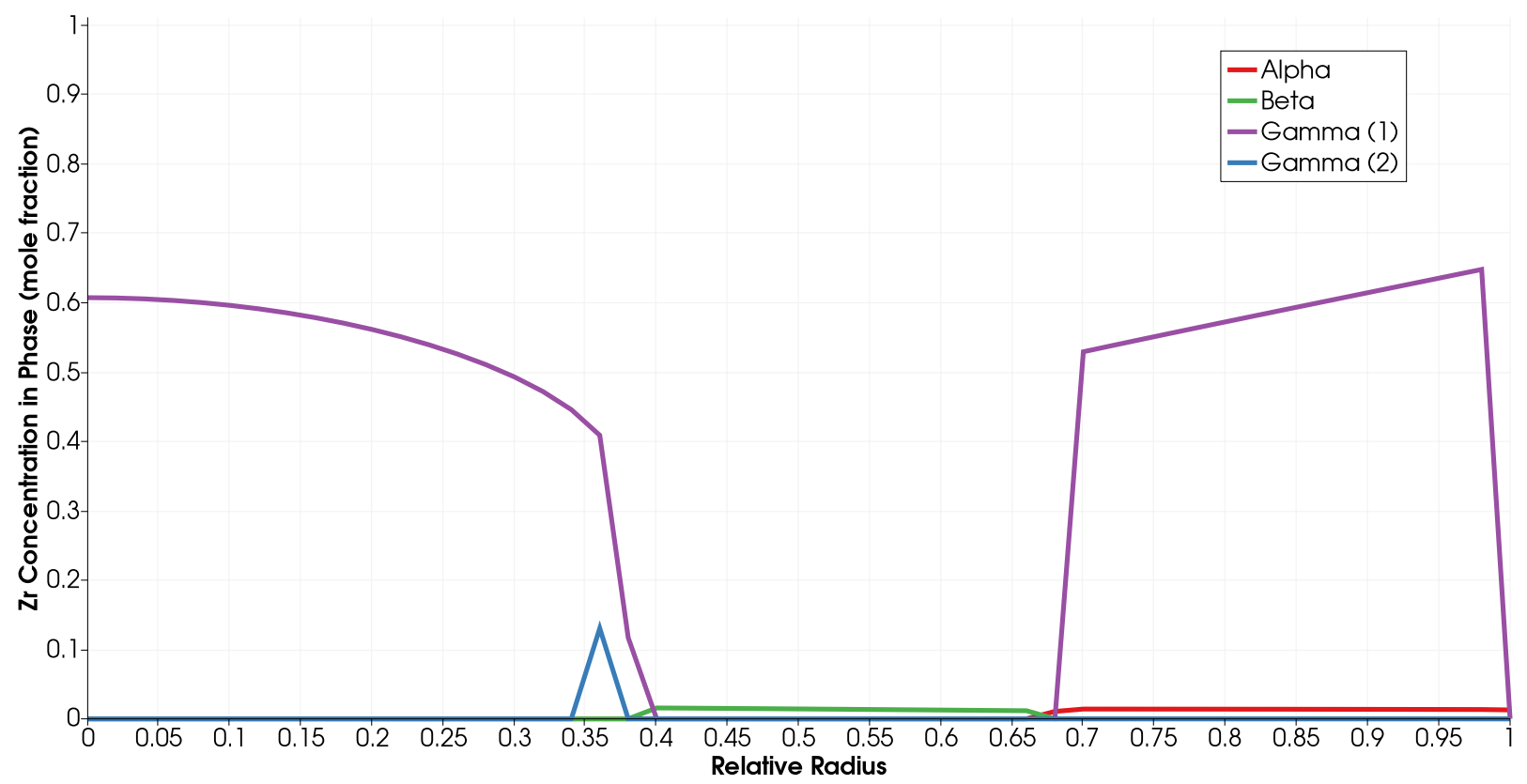

Figure 10. $\mathrm{Zr}$ concentration in each phase for the U-Zr fuel after 50 years of diffusion under the imposed temperature profile with the majority phase rule applied to two-phase regions. 


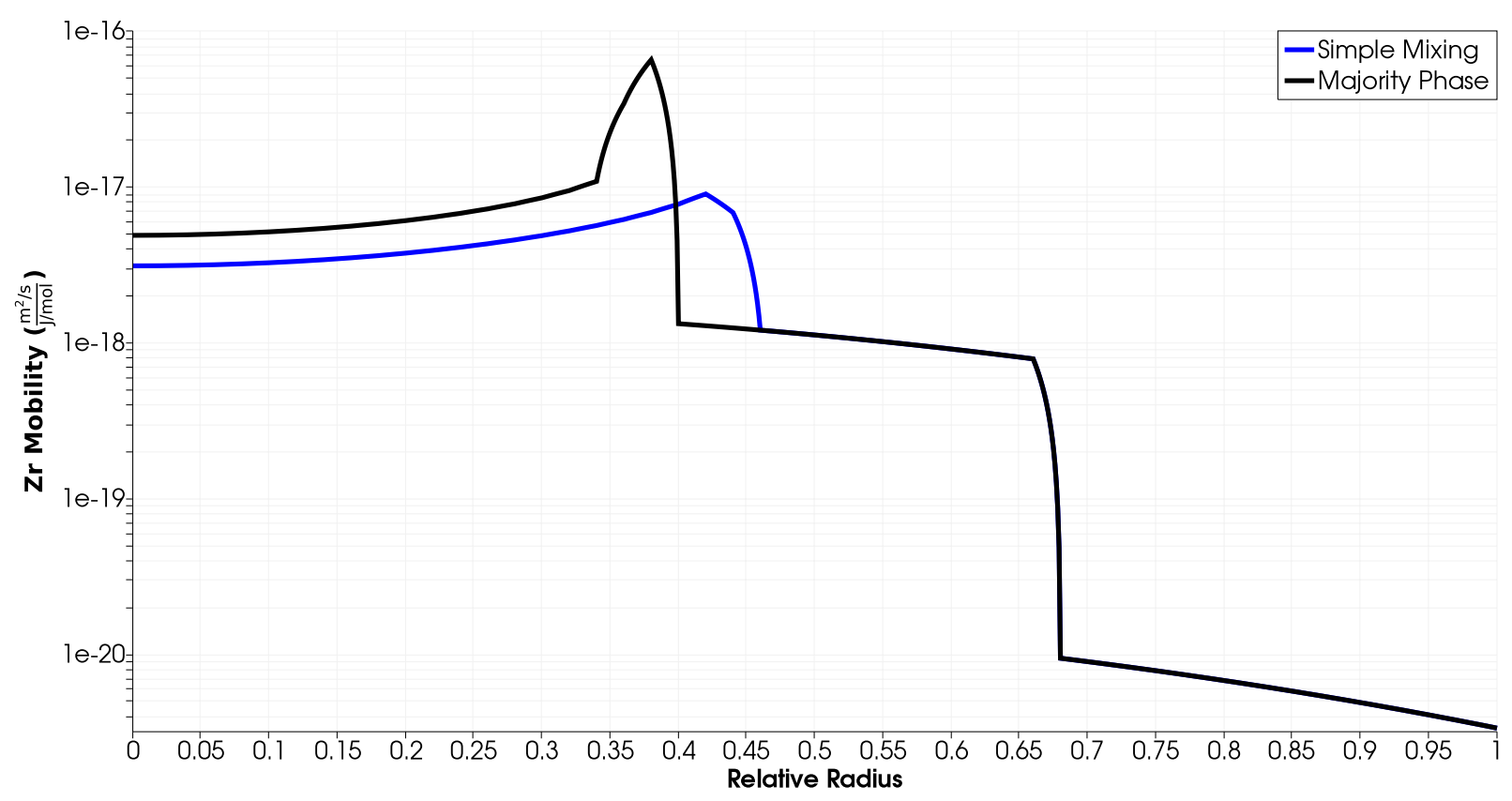

Figure 11. Zr mobility for the fuel element state after 50 years of diffusion under the imposed temperature gradient, as calculated by the simple mixing mobility model and the majority phase mobility model.

\section{CONCLUSION}

The zirconium concentration profiles that evolve after long diffusion times are significantly different for the two models of mobility in two-phase regions employed in this study. Most notably, when the majority phase rule is used the $\mathrm{Zr}$ concentration through most outermost $0.5 \mathrm{~mm}$ of the element remains near the initial value of $22 \mathrm{at} \%$, but when the simple mixing rule is employed the zirconium concentration in this region drops to near zero. Comparing these two models to the experimental results shown in Fig. 1, the majority phase rule does a much better job of reproducing the observations of very little $\mathrm{Zr}$ diffusion in the element exterior, depletion of $\mathrm{Zr}$ in the middle region, and $\mathrm{Zr}$ accumulation toward the center of the element. This highlights the importance of choosing an appropriate model for mobility in two-phase regions.

The presented calculations harnessed the coupled Thermochimica-BISON code system, making use of newly-implemented classes in BISON to obtain equilibrium thermochemical properties such as phase fractions and chemical potential from Thermochimica, and determine relevant thermophysical properties such as mobility and driving force for diffusion. These new classes include a diffusion Kernel that takes mobility input at node locations from an AuxKernel, which is a general implementation that may have uses in other diffusion problems. These tools make use of the existing tools to evaluate Thermochimica at nodes through an AuxKernel and re-initiate Thermochimica calculations to reduce computational costs.

The success of the majority phase model in reproducing experimental $\mathrm{Zr}$ concentration profiles demonstrates the usefulness of this method based on a driving force proportional to the gradient of the generalized chemical potential. However, there remains significant room for improvement to bring the 
simulation conditions closer to experimental conditions. Fission products were not included in this work, and these (most significantly Pu [18]) may significantly change $\mathrm{Zr}$ driving force and mobility.

Counter-diffusion of heavy elements may also be important, though it is expected that the mobility of $U$ is much lower than that of $\mathrm{Zr}$. The assumption of a fixed temperature profile may also influence the simulation results, though the high thermal conductivity and low temperature gradients in metallic fuels (relative to oxide fuels) likely make related effects minimal in this case.

\section{ACKNOWLEDGEMENTS}

Research was sponsored by the U.S. Department of Energy, Office of Nuclear Energy, Nuclear Energy Advanced Modeling and Simulation Program and Fuel Cycle R\&D Program, under contract DE-AC05-00OR22725 with UT-Battelle, LLC. 


\section{REFERENCES}

1. Y. Adda and A. Kirianenko. Study of self-diffusion in $\alpha$ phase uranium. J. Nuclear Materials, 6, 1962.

2. C. B. Basak. Microstructural evaluation of U-rich U-Zr alloys under near-equilibrium condition. Journal of Nuclear Materials, 416(3):280-287, 2011. ISSN 00223115. doi:

10.1016/j.jnucmat.2011.06.011. URL http://dx.doi.org/10.1016/j.jnucmat.2011.06.011.

3. A. Berche, N. Dupin, C. Guéneau, C. Rado, B. Sundman, and J. Dumas. Calphad thermodynamic description of some binary systems involving u. Journal of nuclear materials, 411(1-3):131-143, 2011.

4. D. C. Crawford, D. L. Porter, and S. L. Hayes. Fuels for sodium-cooled fast reactors: Us perspective. Journal of Nuclear Materials, 371(1-3):202-231, 2007.

5. F. Delage, J. Carmack, C. Lee, T. Mizuno, M. Pelletier, and J. Somers. Status of advanced fuel candidates for sodium fast reactor within the generation IV international forum. Journal of nuclear materials, 441(1-3):515-519, 2013.

6. N. Eliaz and L. Banks-Silis. Chemical potential, diffusion and stress - common confusions in nomenclature and units. Corrosion Reviews, 26(2-3):87-103, 2008. ISSN 0334-6005. doi: 10.1515/corrrev.2008.87.

7. G. Eriksson and W. T. Thompson. A procedure to estimate equilibrium concentrations in multicomponent systems and related applications. Calphad, 13(4):389-400, 1989.

8. J. Galloway, C. Unal, N. Carlson, D. Porter, and S. Hayes. Modeling constituent redistribution in U-Pu-Zr metallic fuel using the advanced fuel performance code BISON. Nuclear Engineering and Design, 286:1-17, 2015. ISSN 00295493. doi: 10.1016/j.nucengdes.2015.01.014. URL http://dx.doi.org/10.1016/j.nucengdes.2015.01.014.

9. J. D. Galloway, C. Unal, and C. Matthews. Constituent redistribution in U-Zr metallic fuel using the advanced fuel performance code BISON. Technical report, Los Alamos National Lab.(LANL), Los Alamos, NM (United States), 2016.

10. D. Gaston, C. Newman, G. Hansen, and D. Lebrun-Grandie. MOOSE: A parallel computational framework for coupled systems of nonlinear equations. Nuclear Engineering and Design, 239(10): 1768-1778, 2009.

11. C. Guéneau, S. Gossé, A. Quaini, N. Dupin, B. Sundman, M. Kurata, T. Besmann, P. Turchi, J.-C. Dumas, E.-C. Corcoran, et al. FUELBASE, TAF-ID databases and OC software advanced computational tools to perform thermodynamic calculations on nuclear fuel materials. In ERMSAR 2015-The 7th European Review Meeting on Severe Accident Research, 2015.

12. A. Guillermet Fernandez. Thermodynamic analysis of the stable phases in the $\mathrm{Zr}-\mathrm{Nb}$ system and calculation of the phase diagram. Zeitschrift für Metallkunde, 82(6):478-487, 1991.

13. J. D. Hales, S. R. Novascone, R. L. Williamson, D. R. Gaston, and M. Tonks. Solving nonlinear solid mechanics problems with the Jacobian-free Newton Krylov method. Computer Modeling in Engineering and Sciences, 84(2):123, 2012. 
14. J. M. Harp, L. Capriotti, and F. Cappia. Baseline postirradiation examination of the afc-3c, afc-3d, and afc-4a experiments. Technical report, Idaho National Lab.(INL), Idaho Falls, ID (United States), 2018.

15. J. Hirschhorn, M. R. Tonks, A. Aitkaliyeva, and C. Adkins. A study of constituent redistribution in U-Zr fuels using quantitative phase-field modeling and sensitivity analysis. Journal of Nuclear Materials, 523:143-156, 2019. ISSN 00223115. doi: 10.1016/j.jnucmat.2019.05.053. URL https://doi.org/10.1016/j.jnucmat.2019.05.053.

16. G. L. Hofman, S. L. Hayes, and M. C. Petri. Temperature gradient driven constituent redistribution in U-Zr alloys. Journal of Nuclear Materials, 227(3):277-286, 1996. ISSN 00223115. doi: 10.1016/0022-3115(95)00129-8.

17. M. Ishida, T. Ogata, and M. Kinoshita. Constituent migration model for U-Pu-Zr metallic fast reactor fuel. Nuclear Technology, 104(1):37-51, 1993. ISSN 00295450. doi: 10.13182/NT93-A34868.

18. D. D. Keiser Jr and M. C. Petri. Interdiffusion behavior in U-Pu-Zr fuel versus stainless steel couples. Journal of nuclear materials, 240(1):51-61, 1996.

19. Y. S. Kim, G. L. Hofman, S. L. Hayes, and Y. H. Sohn. Constituent redistribution in U-Pu-Zr fuel during irradiation. Journal of Nuclear Materials, 327(1):27-36, 2004. ISSN 00223115. doi: 10.1016/j.jnucmat.2004.01.012.

20. N. Kocherginsky and M. Gruebele. Mechanical approach to chemical transport. Proceedings of the National Academy of Sciences of the United States of America, 113(40):11116-11121, 2016. ISSN 0027-8424. doi: 10.1073/pnas.1600866113.

21. K. H. Kumar, P. Wollants, and L. Delacy. Thermodynamic assessment of the ti-zr system and calculation of the nb-ti-zr phase diagram. Journal of alloys and compounds, 206(1):121-127, 1994.

22. M. Kurata. Thermodynamic database on U-Pu-Zr-Np-Am-Fe alloy system I - re-evaluation of U-Pu-Zr alloy system. In IOP Conference Series: Materials Science and Engineering, volume 9, page 012022. IOP Publishing, 2010.

23. X. Liu, Z. Li, J. Wang, and C. Wang. Thermodynamic modeling of the U-Mn and U-Nb systems. Journal of nuclear materials, 380(1-3):99-104, 2008.

24. W. Murphy, W. Beck, F. Brown, B. Koprowski, and L. Neimark. Postirradiation examination of $\mathrm{U}-\mathrm{Pu}-\mathrm{Zr}$ fuel elements irradiated in ebr-ii to 4.5 atomic percent burnup. Technical report, Argonne National Lab., Ill., 1969.

25. A. T. Nelson and P. Demkowicz. Other power reactor fuels. In Advances in Nuclear Fuel Chemistry, pages 215-247. Elsevier, 2020.

26. Oak Ridge National Laboratory. THERMOCHIMICA. https://github.com/ORNL-CEES/thermochimica, 2020.

27. R. J. Perez and B. Sundman. Thermodynamic assessment of the Mo-Zr binary phase diagram. Calphad, 27(3):253-262, 2003.

28. M. Petri and M. Dayananda. Isothermal diffusion in uranium-plutonium-zirconium alloys. Journal of nuclear materials, 240(2):131-143, 1997. 
29. M. H. Piro, S. Simunovic, and T. M. Besmann. Thermochimica user manual v1.0. Technical Report ORNL/TM-2012/576, Oak Ridge National Laboratory (ORNL), Oak Ridge, TN (United States), 2012.

30. M. H. A. Piro and S. Simunovic. Performance enhancing algorithms for computing thermodynamic equilibria. Calphad: Computer Coupling of Phase Diagrams and Thermochemistry, 39:104-110, 2012. ISSN 03645916. doi: 10.1016/j.calphad.2012.09.005.

31. M. H. A. Piro and S. Simunovic. Global optimization algorithms to compute thermodynamic equilibria in large complex systems with performance considerations. Computational Materials Science, 118:87-96, 2016. ISSN 09270256. doi: 10.1016/j.commatsci.2016.02.043.

32. M. H. A. Piro, J. Banfield, K. T. Clarno, S. Simunovic, T. M. Besmann, B. J. Lewis, and W. T. Thompson. Coupled thermochemical, isotopic evolution and heat transfer simulations in highly irradiated UO2 nuclear fuel. Journal of Nuclear Materials, 441(1-3):240-251, 2013. ISSN 00223115. doi: 10.1016/j.jnucmat.2013.05.060.

33. M. H. A. Piro, S. Simunovic, T. M. Besmann, B. J. Lewis, and W. Thompson. The thermochemistry library Thermochimica. Computational Materials Science, 67:266-272, 2013. ISSN 09270256.

34. M. Poschmann, M. Piro, and S. Simunovic. Acceleration of Thermochimica calculations in BISON. Report ORNL/TM-2020/1473, Oak Ridge National Laboratory, 2020.

35. A. Quaini, C. Guéneau, S. Gossé, N. Dupin, B. Sundman, E. Brackx, R. Domenger, M. Kurata, and F. Hodaj. Contribution to the thermodynamic description of the Corium-the U-Zr-O system. Journal of Nuclear Materials, 501:104-131, 2018.

36. S. Rothman, J. Gray Jr, J. Hughes, and A. Harkness. Self-diffusion in beta uranium. Journal of Nuclear Materials, 3(1):72-76, 1961.

37. S. Simunovic and T. M. Besmann. Coupling of thermochemistry solver Thermochimica with MOOSE/BISON. Technical report, Oak Ridge National Laboratory (ORNL), Oak Ridge, TN (United States), 2015.

38. S. Simunovic, T. M. Besmann, and S. L. Voit. Benchmark problem for calculating oxygen potential in high burnup LWR fuel using the Thermochimica module in MOOSE/BISON. Technical report, Oak Ridge National Laboratory (ORNL), Oak Ridge, TN (United States), 2014.

39. S. Simunovic, S. L. Voit, and T. M. Besmann. Oxygen diffusion model using the Thermochimica module in MOOSE/BISON. Technical report, Oak Ridge National Laboratory (ORNL), Oak Ridge, TN (United States), 2014.

40. S. Simunovic, T. M. Besmann, E. Moore, K. T. Clarno, W. Wieselquist, J. W. Mcmurray, and M. Piro. Depletion, chemical reaction and transport in high burnup nuclear fuel. Technical report, Oak Ridge National Lab.(ORNL), Oak Ridge, TN (United States), 2019.

41. S. Simunovic, T. Besmann, J. Moore, M. Poschmann, M. Piro, K. Clarno, J. McMurray, and W. Wieselquist. Modeling and simulation of oxygen transport in high burnup LWR fuel. Journal of Nuclear Materials, 2020.

42. W. R. Smith and R. W. Missen. Chemical reaction equilibrium analysis: Theory and algorithms. Wiley, 1982. 
43. Y. H. Sohn, M. A. Dayananda, G. L. Hofman, R. V. Strain, and S. L. Hayes. Analysis of constituent redistribution in the $\gamma$ (bcc) U-Pu-Zr alloys under gradients of temperature and concentrations. Journal of Nuclear Materials, 279(2-3):317-329, 2000. ISSN 00223115. doi: 10.1016/S0022-3115(99)00290-1.

44. The Nuclear Energy Agency (NEA). Thermodynamics of Advanced Fuels - International Database (TAF-ID). https://www.oecd-nea.org/science/taf-id/, 2020.

45. W. B. White, S. M. Johnson, and G. B. Dantzig. Chemical equilibrium in complex mixtures. The Journal of Chemical Physics, 28(5):751-755, 1958. 